\title{
Use of radionuclide labelled microspheres to show the distribution of the pulmonary perfusion with multifocal pulmonary blood supply
}

\author{
E J BAKER, J MALAMITSI, O D H JONES, M N MAISEY, M J TYNAN \\ From the Departments of Paediatric Cardiology and Nuclear Medicine, Guy's Hospital, London
}

SUMMARY In pulmonary atresia with a ventricular septal defect and similar congenital heart disorders the pulmonary blood supply is often multifocal. The relative distribution of pulmonary perfusion from individual sources is usually demonstrated by selective angiography. A new technique using a selective injection of radionuclide labelled human albumin microspheres was performed in four patients with a complex pulmonary blood supply. In these cases the physiological distribution of each of the multiple sources of pulmonary perfusion was demonstrated. This is a useful additional technique in the assessment of such patients.

In pulmonary atresia with a ventricular septal defect the pulmonary blood supply is often multifocal. ${ }^{1}$ Individual parts of the pulmonary vasculature may be connected to the central pulmonary arteries, may be supplied directly by major aortopulmonary collaterals, or may have a dual blood supply. ${ }^{2}$ The central pulmonary arteries are often connected to less than half the bronchopulmonary segments. ${ }^{3}$ A knowledge of the precise nature of the pulmonary blood supply in an individual patient is essential for the rational surgical management. 34

Conventionally, the anatomy of the pulmonary circulation is demonstrated by selective angiography. ${ }^{15}$ Although this technique shows the anatomy of the arterial supply very well, it gives limited information on the true physiological blood flow, especially in the presence of a duplicate blood supply. ${ }^{2}$ Pressure injections of contrast medium through catheters in arteries have been shown to cause a substantial increase in the flow during the period of the injection. ${ }^{6}$ This effect means that the observed pattern of blood flow cannot be assumed to represent the physiological distribution.

In contrast, the injection of radionuclide labelled microspheres offers the possibility of studying the pulmonary blood flow without haemodynamic disturbance. We investigated the possibility of this

Requests for reprints to Dr E J Baker, Department of Paediatrics, Guy's Hospital, London SE1 9RT.

Accepted for publication 14 February 1984 approach with a new technique to demonstrate the distribution of the pulmonary blood supply by selective injection of radionuclide labelled human albumin microspheres in patients with a complex pulmonary blood supply.

\section{Patients and method}

Radionuclide labelled human albumin microspheres were injected at selected sites in the circulation during a full haemodynamic and angiographic assessment in four patients with a complex pulmonary blood supply. Dilute contrast medium was injected by hand before the microspheres were given to ensure the catheter position did not encourage preferential streaming. The microspheres $(3 \mathrm{M}$, instant microspheres) were given in a volume of approximately 0.5 $\mathrm{ml}$ and were flushed in with $5 \mathrm{ml}$ of saline. They have a mean diameter of $20 \mu \mathrm{m}$ with a standard deviation of $5 \mu \mathrm{m}$. In three cases they were labelled with technetium- $99 \mathrm{~m}$ alone. In case 2 indium- $113 \mathrm{~m}$ labelled microspheres were also used so that injections could be made at two separate sites in the central circulation. The different energies of the gamma rays emitted by these two radionuclides, $140 \mathrm{keV}$ for technetium-99m and $392 \mathrm{keV}$ for indium-113m, allow the distribution of microspheres from each injection to be imaged independently. A high energy collimator is required for the indium image.

Static images of the lungs were acquired for five minutes after each injection. In cases 3 and 4 a mobile 
gammacamera was used in the catheter laboratory. In these two studies multiple injections of technetium labelled microspheres were given at selected sites in the central circulation. Anterior lung images were acquired after each injection. The patients were positioned identically for each image with two cobalt-57 marker sources attached to their chests. The images were stored on magnetic tape and later analysed on a digital computer. The distribution of microspheres from each injection was reconstructed by subtraction of these stored images.

The total number of microspheres given in each case was $30000 / \mathrm{m}^{2}$. This represents approximately $0.3 \mathrm{mg}$ of human serum albumin. The total dose of technetium in each case was $64 \mathrm{MBq} / \mathrm{m}^{2}$ and of indium (in case 2) $21 \mathrm{MBg} / \mathrm{m}^{2}$. When multiple injections of the technetium microspheres were used the total dose was divided into the appropriate number of aliquots.

\section{CASE REPORTS}

Case 1

A 9 year old girl with pulmonary atresia and a ventricular septal defect had a palliative reconstruction of the right ventricular outflow tract. She was known to have two major collateral arteries arising from the descending aorta supplying parts of both the right and left lungs. Three years later she was requiring treatment for cardiac failure and was reinvestigated.

An injection of technetium-99m labelled microspheres into the pulmonary artery showed a uniform distribution throughout the right lung, whereas a large part of the left lung was not perfused. The central pulmonary artery supplied only part of the left upper and lower lobes. The whole of the left lung was perfused when a second injection of technetium labelled microspheres was given into a peripheral vein on a separate occasion. The collateral artery supplying the left lung was therefore the sole supply to a large part of the pulmonary vascular bed, whereas the right lung had a dual blood supply from both the central pulmonary artery, which had been shown to supply all parts of the lung, and the known right collateral artery. The right collateral artery was since surgically ligated and the heart failure clinically responded.

\section{Case 2}

A 13 month old child with pulmonary atresia and a ventricular septal defect was investigated. He had a right Blalock-Taussig shunt created at 1 week of age. At cardiac catheterisation the shunt was entered and appeared to be the sole blood supply to the lungs on angiography. Indium-113m microspheres were injected into the Blalock-Taussig shunt and technetium-99m microspheres into the root of the aorta. Static images were then acquired for the two isotopes. The quality of the image with the indium labelled microspheres was poorer than that obtained with the technetium label. Both images, however, showed preferential blood flow to the right lung with no difference in the distribution of perfusion. The ratio of right lung activity to left lung activity for the indium image was 1.4:1 and for the technetium image 1.6:1. Thus there was no evidence of any separate collateral supply to either lung.

\section{Case 3}

A 14 year old boy with pulmonary atresia and a ventricular septal defect had had a palliative reconstruction of the right ventricular outflow tract one year previously. He was known to have multiple aortopulmonary collaterals. Technetium-99m labelled microspheres were injected into the pulmonary artery and an anterior image acquired using a mobile gammacamera. The majority of the blood flow from the central pulmonary arteries $(65 \%)$ was to the left lung with only $35 \%$ of the microspheres distributed to parts of the right lung (Figure a). A further injection of the technetium microspheres was given into a major collateral arising from the left subclavian artery and a second image acquired. The distribution of the blood flowing through the collateral was obtained by subtracting the first image from the second (Figure b). A third injection of microspheres was given into a collateral arising from the descending aorta, supplying some of the right lung. Subtraction was again used to show the distribution of the blood flow (Figure c). The final image acquired (Figure d) showed that all parts of the lungs were perfused from these three sources.

\section{Case 4}

In a 22 year old man with the tetralogy of Fallot and an anomalous origin of the left pulmonary artery from the ascending aorta the very small pulmonary artery arising from the right ventricle supplied only the right lung.

An injection of technetium microspheres was given into the left pulmonary artery at its origin and an image acquired. There was uniform perfusion of the left lung but none to the right. The right pulmonary artery could not be entered directly so a second injection was given into the body of the right ventricle. The image obtained by subtraction demonstrating the distribution of flow from the right ventricle showed that the majority of the blood flow was to the left lung. Despite the right pulmonary artery arising directly from the right ventricle only $18 \%$ of the flow from the ventricle was to the right lung. A further injection was given into the aorta just distal to the left pulmonary artery. The image obtained showed no additional areas of lung perfused, so there could be no other 


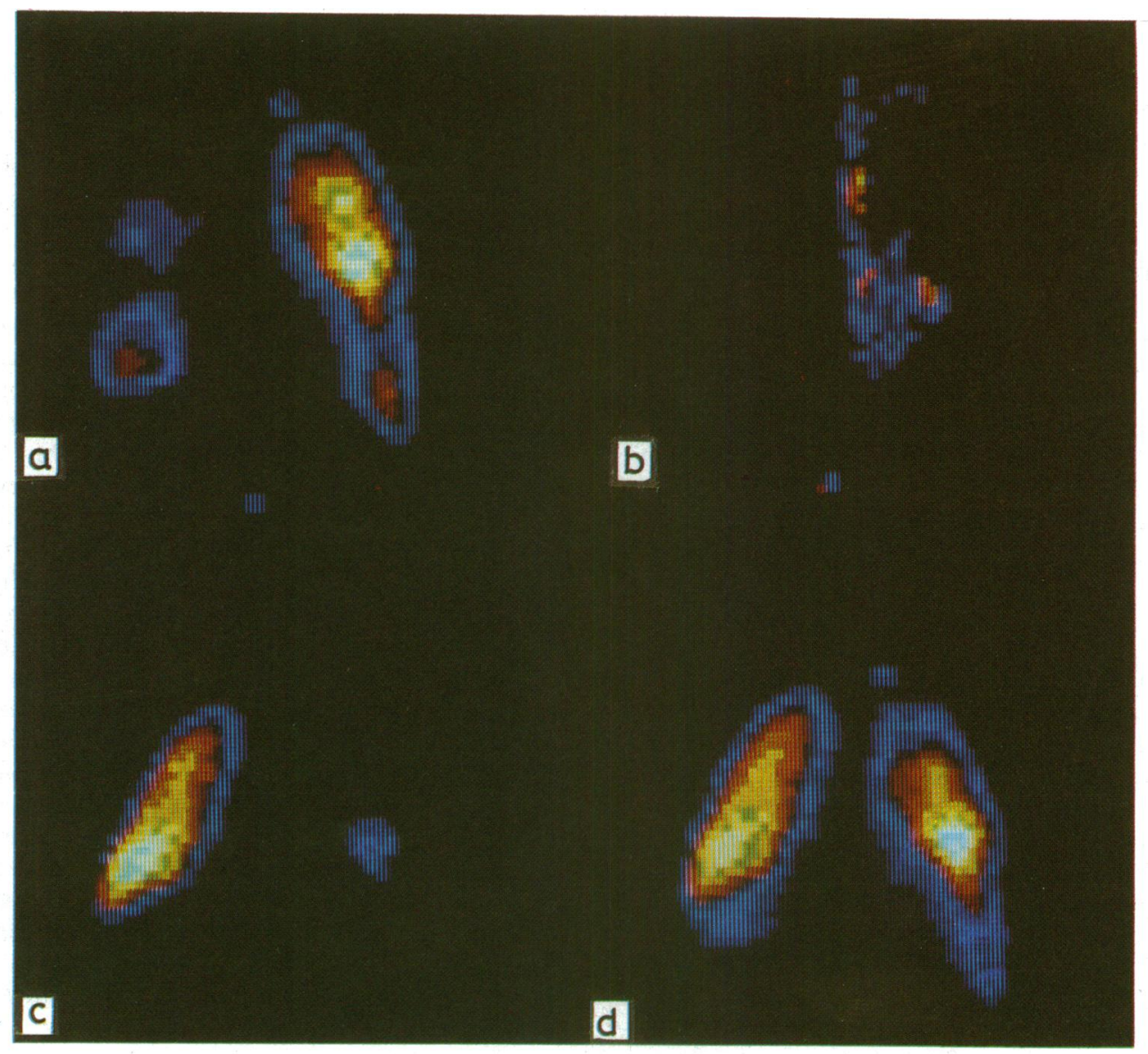

Figure Radionuclide anterior images in case 3: (a) image after pulmonary artery injection; (b) subtracted image to show the distribution of microspheres after injection into the left collateral; $(c)$ subtracted image after injection into the right collateral; and (d) final image obtained after all three injections.

significant pulmonary blood supply from collateral arteries arising distal to the left pulmonary artery.

\section{Discussion}

Radionuclide labelled human albumin microspheres have been shown to be an effective method of demonstrating regional pulmonary blood flow. ${ }^{78}$ Microspheres of the size used here can be expected to lodge in approximately $0.1 \%$ of the pulmonary capillaries, 9 and only a very small proportion of the radionuclide label escapes capture in the pulmonary circulation. ${ }^{1011}$ The injection of such microspheres into the pulmonary, cerebral, and coronary circulations has been shown to be safe. ${ }^{12-14}$ In clinical use no major complication except for rare anaphylactoid reaction has been reported. ${ }^{15}$
Radionuclide labelled macroaggregated albumin has been used to measure the magnitude of right to left shunting in congenital heart disease ${ }^{16}$ and also to study lung perfusion after corrective surgery. ${ }^{17}$ The selective injection of microspheres to demonstrate the distribution of pulmonary perfusion in a complex pulmonary vascular supply has, however, not been reported.

In the present four cases microspheres proved to be valuable in showing the distribution of perfusion from individual vessels supplying the pulmonary circulation. The volume of fluid given is small and injected by hand, so the distribution is likely to reflect the true physiological perfusion unlike that after pressure injections of contrast media. ${ }^{6}$ The correspondence of the relative flow to the right and left lungs between the aortic injection and the injection into the surgical 
shunt in case 2 supports the assumption inherent in this technique that direct injection into a supply artery gives a true picture of the distribution of its blood flow and that streaming of the microspheres was not a problem. Digital subtraction of images enables multiple injections to be given at several sites and the distribution of the microspheres from each individual injection to be established. Alternatively, as in case 2, microspheres labelled with different radionuclides can be used.

It must be emphasised that the images from the microsphere studies can be interpreted only in the light of the known vascular anatomy in each case, as defined by angiography. Case 1 showed the limited distribution of the central pulmonary artery perfusion in a case of pulmonary atresia with a ventricular septal defect. The left collateral artery was shown to supply a substantial part of the left lung, whereas the right lung had a dual blood supply from the central pulmonary artery and the right collateral artery. In case 2 the microsphere study showed the absence of any significant pulmonary perfusion additional to the surgical shunt and therefore excluded any major aortopulmonary collateral arteries. In case 3 the pulmonary artery supplied the majority of the left lung but only the minority of the right lung (Figure a). The remainder of the pulmonary circulation was perfused from two separate systemic-pulmonary collateral arteries (Figure $b$ and $c$ ). There was no signficant overlap between these three distributions. In case 4 the vessel arising from the ascending aorta perfused the whole of the left lung, and the vessel arising from the right ventricle perfused the right lung only poorly. There was no other source of blood flow to the right lung.

There are several problems with this technique. Indium-113m has a half life of only 1.73 hours, and, although the escape of radionuclide from the pulmonary capillaries is negligible immediately after the injection, it becomes considerable if there is a delay before the image is acquired. ${ }^{10}$ For both these reasons it is important that images are acquired as soon as possible after the microspheres have been given. Delay will diminish the quality of the images obtained. This consideration led us to use a mobile gammacamera in the catheter laboratory, and digital subtraction enabled us to give multiple injections of one isotope, technetium- $99 \mathrm{~m}$, in place of differently labelled microspheres.

Digital subtraction requires that the second image obtained exactly superimposes on the first. We took great care with positioning patients, using marker sources attached to their chests, and performed the acquisitions as soon after one another as feasible to reduce the risk of movement. This also reduces the effect of any radioactive decay or escape of the radionuclide from the pulmonary capillaries between injections.

An alternative approach to overcome the problems we encountered using indium- $113 \mathrm{~m}$ would be to use a second radionuclide with a longer half life, for instance indium-111. ${ }^{11}$ The dual radionuclide method limits the number of sites at which injections can be made to two, whereas the subtraction method allows as many injections to be given as necessary to suit the anatomy in each case. We have successfully given a maximum of three separate injections of microspheres using this technique. Nevertheless, a mobile gammacamera must be used, and only the anterior projection is feasible. Oblique and posterior images would improve the definition of the distribution of perfusion. These can be obtained only with the dual radionuclide technique.

Although in case 2 we were able to establish that streaming of the microspheres preferentially into one of the pulmonary arteries was not a problem, this cannot be assumed in every case. Careful positioning of the catheter tip using small contrast injections is essential if this problem is to be avoided.

The selective injection of radionuclide labelled human albumin microspheres in conditions such as pulmonary atresia with a ventricular septal defect gives a good visual assessment and some semiquantitative information on the distribution of the pulmonary blood flow. This is a potentially valuable additional technique in the preoperative assessment of conditions with a complex pulmonary blood supply.

E J B is a British Heart Foundation junior research fellow.

\section{References}

1 Macartney FJ, Scott O, Deverall PB. Haemodynamic and anatomical characteristics of pulmonary blood supply in pulmonary atresia with ventricular septal defect, including a case of persistent fifth aortic arch. Br Heart $\mathcal{f}$ 1974; 36: 1049-60.

Z Fäller K, Haworth SG, Taylor JFN, Macartney FJ. Duplicate sources of pulmonary blood supply in pulmonary atresia with ventricular septal defect. Br Heart $\mathcal{F}$ 1981; 46: 263-8.

3 Haworth SG, Macartney FJ. Growth and development of pulmonary circulation in pulmonary atresia with ventricular septal defect and major aortopulmonary collateral arteries. Br Heart f 1980; 44: 14-24.

4 McGoon DC, Baird DK, Davis GD. Surgical management of large bronchial collateral arteries with pulmonary stenosis or atresia. Circulation 1975; 52: 109-18.

5 Soto B, Pacifico AD, Luna RF, Bargeron LM Jr. A radiographic study of congenital pulmonary atresia with ventricular septal defect. AfR 1977; 129: 1027-37. 
6 Levin DC. Augmented arterial flow and pressure resulting from selective injections through catheters: clinical implications. Radiology 1978; 127: 103-8.

7 Wagner HN Jr, Sabiston DC Jr, lio M, McAfee JG, Meyer JK, Langan JK. Regional pulmonary blood flow in man by radioisotope scanning. $\mathcal{F} A M A$ 1964; 187: 601-3.

8 Rhodes BA, Zolle I, Buchanan JW, Wagner HN Jr. Radioactive albumin microspheres for studies of the pulmonary circulation. Radiology 1969; 92: 1453-60.

9 Harding LK, Horsfield K, Singhal SS, Cumming G. The proportion of lung vessels blocked by albumin microspheres. I Nucl Med 1973; 14: 579-81.

10 Toombs BD, McKusick KA, Castronovo FP, Callahan RJ, Stauss HW. Urinary excretion of ${ }^{99 \mathrm{~m}} \mathrm{Tc}$ administered as microspheres. Radiology 1980; 134: 489-91.

11 Hagan PL, Krejcarek GE, Taylor A, Alazraki N. A rapid method for the labelling of albumin microspheres with In-113 and In-111: concise communication. 7 Nucl Med
1978; 19: 1055-8.

12 Allen DR, Ferens JM, Cheney FW, Nelp WB. Critical evaluation of acute cardiopulmonary toxicity of microspheres. F Nucl Med 1977; 19: 1204-8.

13 Verhas M, Schoutens A, Demol O, et al. Use of ${ }^{{ }^{9 m} \mathrm{~m}} \mathrm{Tc}$ labeled albumin microspheres in cerebral vascular disease. $f$ Nucl Med 1976; 17: 170-4.

14 Grames GM, Jansen C, Gander MP, Wieland HC, Judkins MP. Safety of the direct coronary injection of radiolabeled particles. $\mathcal{F}$ Nucl Med 1974; 15: 2-6.

15 Littenberg RL. Anaphylactoid reaction to human albumin microspheres. $\mathcal{F}$ Nucl Med 1975; 16: 236-7.

16 Gates GF, Orme HW, Dore EK. Measurement of cardiac shunting with Technetium labeled albumin aggregates. I Nucl Med 1971; 12: 746-9.

17 Alderson PO, Boonvisut S, McKnight RC, Hartman AF Jr. Pulmonary perfusion abnormalities and ventilationperfusion inbalance in children after total repair of tetralogy of Fallot. Circulation 1976; 53: 332-7. 